\title{
Dirofilaríase pulmonar humana no Estado do Rio de Janeiro, Brasil: relato de um caso
}

\author{
Human pulmonary dirofilariasis at \\ Rio de Janeiro, Brazil: a case report
}

\author{
Rosângela Rodrigues-Silva ～', Rodrigo Jorge de Alcantara Guerra ～' , Fernanda Barbosa de Almeida ～ \\ José Roberto Machado-Silva ${ }^{2}$ e Daurita Darci de Paiva ${ }^{3}$
}

\section{RESUMO}

Relata-se caso de dirofilaríase pulmonar humana em paciente com 45 anos de idade, proveniente do Estado do Rio de Janeiro, Brasil. A radiografia torácica evidenciou nódulo pulmonar solitário localizado no lóbulo inferior esquerdo, mimetizando neoplasia. Dirofilaríase pulmonar humana deveria ser investigada em nódulos pulmonares não malignos e de etiologia não conclusiva.

Palavras-chaves: Dirofilaríase pulmonar humana. Dirofilaria immitis. Nódulo pulmonar solitário. Brasil.

\section{ABSTRACT}

We report a case of human pulmonary dirofilariasis in a female patient 45-years-old, derived from Rio de Janeiro, Brazil. The chest radiologic finding consisted of single pulmonary nodule located on the left lower lobe that simulated lung tumor. Human pulmonary dirofilariasis might be investigated in nodules that are not malignants and require conclusive diagnosis.

Key-words: Human pulmonary dirofilariasis. Dirofilaria immitis. Solitary pulmonary nodule. Brazil.

Dirofilaríase pulmonar humana (DPH) é uma doença zoonótica, cuja apresentação mais comum é um nódulo pulmonar solitário que mimetiza câncer de pulmão ${ }^{9}$. As microfilárias do agente etiológico Dirofilaria immitis transmitidas por mosquitos dos gêneros Culex, Anopheles e Aedes, morrem no coração e são conduzidas para o tecido pulmonar, através da artéria pulmonar, onde geram quadros de embolia pulmonar ${ }^{534}$.

No tecido pulmonar, as larvas induzem a formação de nódulos não calcificados, normalmente não produzindo ameaça à saúde do homem por apresentarem uma evolução benigna. Devido à semelhança radiológica entre o nódulo e o tumor pulmonar primário ou metastático, o diagnóstico laboratorial requer a aplicação de recursos invasivos como a toracotomia ou a biópsia pulmonar ${ }^{6715273134}$. Como muitas vezes os nódulos pulmonares não são malignos e não apresentam um diagnóstico conclusivo $^{2839}$, é possível que casos de DPH estejam sendo subdiagnosticados.

A freqüência e a distribuição da dirofilaríase pulmonar humana e canina em uma área geográfica são influenciadas pelo tamanho da população canina, pela prevalência da infecção nestes animais, pela densidade dos mosquitos vetores e pelo nível de exposição do homem à picada dos mosquitos infectados ${ }^{11}$. Ainda que a infecção canina tenha ampla distribuição geográfica, ocorre com maior freqüência em áreas litorâneas ${ }^{2122}$. Embora haja uma alta ocorrência de infecção canina no Brasil, a sua distribuição não está completamente conhecida ${ }^{54}, \mathrm{com}$ possibilidade que não seja restrita às áreas litorâneas. Através de um inquérito soro-epidemiológico realizado em cães na cidade de Cuiabá (MT), constatou-se que 9,6\% eram positivos ${ }^{18}$.

Embora no Brasil, a DPH seja pouco relatada (Tabela 1), o maior número de relatos concentra-se na região sudeste (São Paulo e Rio de Janeiro $)^{625} 3334$. Recentemente, entretanto foram relatados oito casos humanos em Santa Catarina ${ }^{79}$.

\section{RELATO DO CASO}

Paciente do sexo feminino, 45 anos, doméstica, natural e moradora do Rio de Janeiro foi atendida no Hospital Universitário

\footnotetext{
1. Laboratório de Helmintos Parasitos de Vertebrados do Departamento de Helmintologia do Instituto Oswaldo Cruz da Fundação Oswaldo Cruz, Rio de Janeiro, RJ. 2. Disciplina de Parasitologia e 3. Disciplina de Anatomia Patológica do Departamento de Patologia e Laboratórios da Faculdade de Ciências Médicas da Universidade do Estado do Rio de Janeiro, Rio de Janeiro, RJ.

Endereço para correspondência: Dra. Rosângela Rodrigues e Silva. Av. Brasil 4365. Dept ${ }^{\circ}$ de Helmintologia/FIOCRUZ. Av. Brasil 4365, Manguinhos, $21045-900$ Rio de Janeiro, RJ

Telefax: 5521 2598-4363

e-mail: rsilva@ioc.fiocruz.br

Recebido para publicação em 25/03/2003

Aceito em: 5/12/2003
} 
Tabela 1 - Casos de dirofilariase pulmonar bumana no Brasil.

\begin{tabular}{|c|c|c|}
\hline Localidade & $\operatorname{Casos}\left(\mathrm{n}^{\circ}\right)$ & Referência \\
\hline Rio de Janeiro & 1 & Magalhães ${ }^{26}$ \\
\hline Indeterminado* & 1 & Leonardi et $_{a l^{24}}$ \\
\hline São Paulo & 1 & Schneider et $^{3} \mathrm{l}^{36}$ \\
\hline Rio de Janeiro & 3 & Madi $e t a l^{25}$ \\
\hline São Paulo & 1 & Saad $e t a l^{35}$ \\
\hline São Paulo & 9 & Barbas Filho et al \\
\hline São Paulo & 1 & Amato Neto et $a l^{l}$ \\
\hline São Paulo & 1 & Amato et al ${ }^{1}$ \\
\hline São Paulo & 24 & Campos et al ${ }^{6}$ \\
\hline Santa Catarina & 7 & Cavallazzi $e t a l^{9}$ \\
\hline Santa Catarina & 1 & Cardoso et al \\
\hline Total & 50 & \\
\hline
\end{tabular}

* Paciente residente no Brasil e diagnosticado nos EUA.

Pedro Ernesto com constantes dores torácicas. A radiografia de tórax evidenciou um nódulo pulmonar solitário localizado no lóbulo inferior esquerdo. Foi realizada uma toracotomia exploratória que apresentou o seguinte laudo: 1) macroscopia: fragmento irregular de pulmão medindo 4 x $3,5 \mathrm{~cm}$, parcialmente recoberto por pleura lisa e brilhante; aos cortes, área nodular com limites imprecisos de coloração parda acinzentada, com áreas esbranquiçadas e de consistência algo friável; 2) microscopia (congelação): processo inflamatório crônico, com ausência de neoplasia.

0 fragmento de pulmão foi então fixado em formalina tamponada de Carson's pH 7, $4^{8}$ e incluído em parafina. Os cortes (5mm) foram corados com hematoxilina e eosina (HE), GiemsaLennert ${ }^{23}$ e tricrômico de Masson.

0 resultado histopatológico revelou áreas de infarto e abscesso, com reação gigantocitária, centrado por helminto compatível com Dirofilaria immitis, infiltrado inflamatório mono e polimorfonuclear, com acentuado predomínio de eosinófilos (Figuras 1A, B e C).

\section{DISCUSSÃ0}

Animais de estimação oferecem significantes benefícios aos indivíduos e à comunidade. Entretanto, deve-se ter o conhecimento do potencial de transmissão dos agentes parasitários destes animais para 0 homem ${ }^{32}$. Dirofilaria immitis é um nematódeo que, na fase adulta, habita o ventrículo direito de cães e gatos, enquanto as microfilárias são transmitidas para os mamíferos durante o hematofagismo. Eventualmente, o homem pode ser picado por um inseto infectado. Neste caso, por ser o homem um hospedeiro inadequado para o parasito, as microfilárias morrem no coração e são conduzidas para o tecido pulmonar através da artéria pulmonar ${ }^{53}$.

A dirofilaríase pulmonar humana é um problema complexo de importância em saúde pública e medicina preventiva, envolvendo principalmente seu diagnóstico diferencial com um tumor pulmonar primário ou metastático. Com freqüência, o encontro destes nódulos pulmonares decorre da realização de exame radiológico de tórax e posterior necessidade de elucidação etiológica ${ }^{2} 38.0$ diagnóstico requer a aplicação de recursos invasivos como a toracotomia ou a biópsia pulmonar ${ }^{3134}$. Distintos estudos mostram que a maioria dos
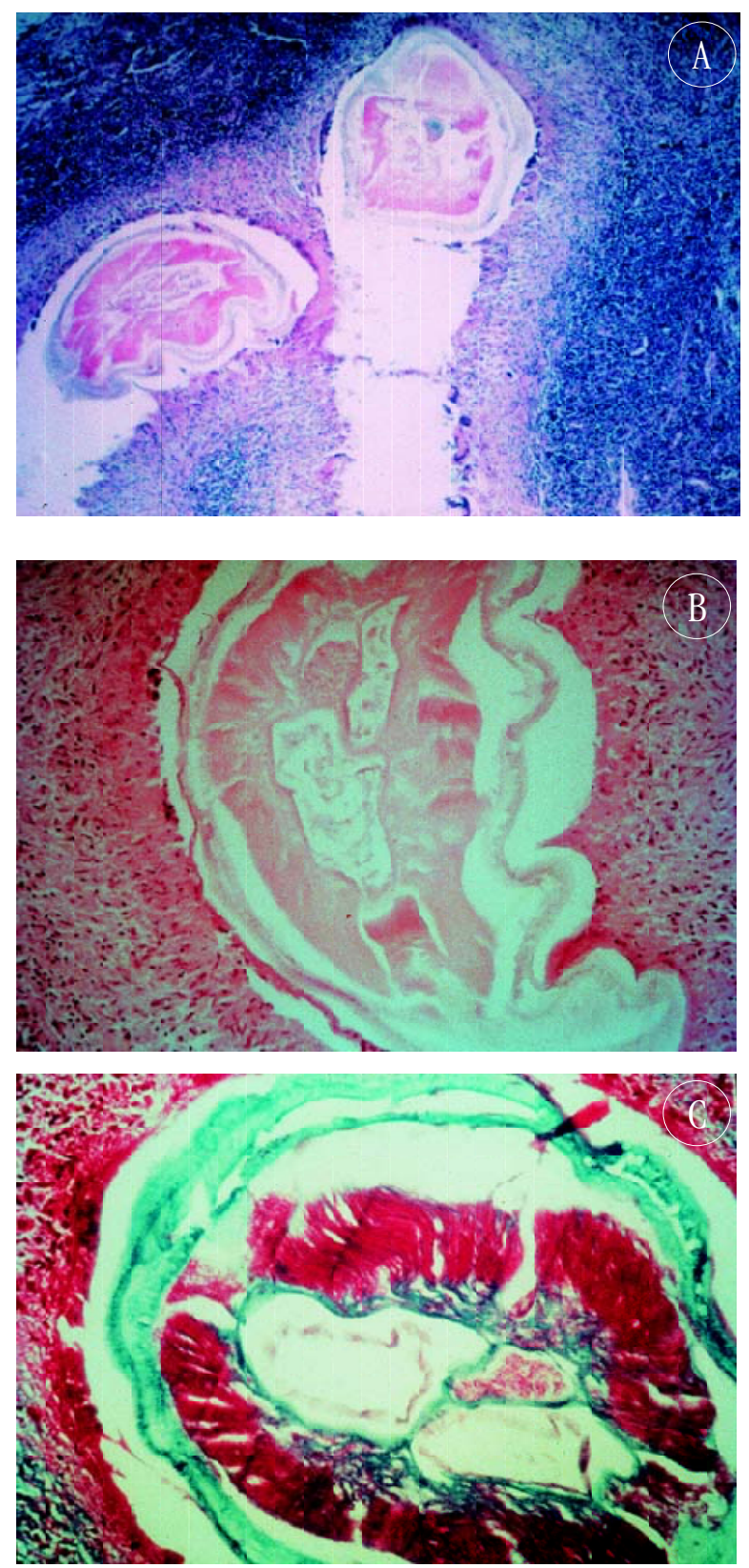

Figura 1 - Cortes histológicos mostrando área de infarto pulmonar centrado por belminto compativel com Dirofilaria immitis. A: Giemsa (100X); B: Hematoxilina-eosina (160X);C: Tricômico de Masson, (200X).

casos de DPH é de pacientes assintomáticos detectados em exames radiológicos de rotina ou direcionados à outras lesões pulmonares ${ }^{6931}$. Nos indivíduos com sintomatologia, destaca-se: a dor torácica, tosse, hemoptise, dor na garganta, sibilo, calafrio, febre, dispnéia dependente de esforço, fadiga, síncope e emagrecimento 24101113141619 .

Quanto ao presente caso, alguns pontos podem ser destacados: tanto a faixa etária quanto o sexo diferem de outros autores que citam maior ocorrência de DPH no sexo masculino e acima dos 50 anos de idade ${ }^{631}$. Uma possível explicação para estas observações associa a imunidade de crianças e adolescentes com a baixa ocorrência nestas faixas, diferenças hormonais entre os sexos e um 
maior número de radiografias de tórax realizadas por pacientes do sexo masculino ${ }^{631}$.

A dor torácica como queixa principal é assinalada tanto em pacientes diagnosticados nos Estados Unidos ${ }^{31}$ quanto em diferentes cidades (São Paulo e Florianópolis) do Brasil ${ }^{69}$. Ainda que a questão de ocorrência de diferentes cepas em $D$. immitis não seja descrito, a sintomatologia em humanos parece não ser influenciada por este aspecto. Entre os 24 casos de DPH analisados em São Paulo, os nódulos solitários estavam situados mais no lobo superior direito $(33,5 \%)$ e quando no esquerdo, era no lobo inferior $(21 \%)^{6}$. Nossos dados concordam com estas observações. Não foi possível obter dados quanto ao contato da paciente com animais domésticos e tabagismo.

Ainda que a dirofilaríase tenha sido descrita no Brasil por Magalhães em $1887^{26}$ até agora só foram descritos e confirmados 50 casos (exceto o presente). É sabido que nem todos os nódulos pulmonares solitários são diagnosticados como neoplasias, portanto, a hipótese de dirofilaríase pulmonar deve ser considerada. Entretanto, a ausência de um diagnóstico parasitológico (o parasito não completa o ciclo no homem), de uma efetiva sorologia (só é positiva quando o nódulo ainda está em formação) e a necessidade de confirmação por exame anatomopatológico, sugerem que esta zoonose seja subdiagnosticada em nosso meio.

A reação em cadeia da polimerase (PCR), que permite a amplificação de regiões do genoma a partir de mínimas quantidades de DNA, mesmo degradado, é uma metodologia de escolha para a utilização de tecido fixado em formalina e incluído em blocos de parafina $^{12} 172937$. Recentemente, desenvolvemos um diagnóstico molecular para DPH baseado na seqüência do espaçador ribossomal ITS 2, o que torna possível estudos retrospectivos desta patologia ${ }^{20}$. Estes tecidos emblocados estão disponíveis em Serviços de Patologia, o que permite ainda estudos retrospectivos, constituindo uma vasta fonte de pesquisa ${ }^{2930}$.

0 aumento da população canina, as condições favoráveis para a proliferação de mosquitos e o seu hematofagismo favorecem 0 aparecimento de novos casos de dirofilaríase humana. Por estas razões, clínicos, radiologistas e patologistas devem estar atentos aos nódulos pulmonares solitários sem características de neoplasia, considerando-se a DPH como diagnóstico diferencial de nódulos pulmonares $^{6} 3436$

\section{REFERÊNCIAS BIBLIOGRÁFICAS}

1. Amato VS, Amato-Neto V, Uip DE, Boulos M. Novo caso de dirofilariose pulmonar humana adquirida no Brasil. Revista da Sociedade Brasileira de Medicina Tropical 28: 285-286, 1995.

2. Amato Neto V, Amato VS, Moraes Jr ACP, Cerri GG. Dirofilariose pulmonar humana, adquirida no Brasil: comunicação de um caso. Revista do Instituto de Medicina Tropical de São Paulo 35: 457-460, 1993.

3. Barbas Filho JV, Barbas CSV, Tucci MR, Jatene F, Milanez JR, Curi N. Human pulmonary dirofilariasis: study of nine cases. American Review of Respiratory Disease 145 (supl): A550, 1992

4. Beskin CA, Colvin SH, Beaver PC. Pulmonary dirofilariasis. Cause of pulmonary nodular disease. Jounal of the American Medical Association 198: 665-667, 1966.
5. Brito AC, Vila-Nova MC, Rocha DAM, Costa LG, Almeida WAP, Viana LS, Lopes Jr RR, Fontes G, Rocha EMM, Regis L. Prevalência da filariose canina causada por Dirofilaria immitis e Dipetalonema reconditum em Maceió, Alagoas, Brasil. Cadernos de Saúde Pública 17: 1497-1504, 2001.

6. Campos JRM, Barbas CSV, Filomeno LTB, Fernandez A, Minamoto H, Barbas Filho JV, Jatene FB. Human Pulmonary Dirofilariasis. Analysis of 24 cases from São Paulo, Brazil. Chest 112: 729-733, 1997.

7. Cardoso JJ, Silva FM, Silveira RJ, Valle AD, Nobre LF, Souza IV. Múltiplos nódulos pulmonares - Dirofilariose. Jornal de Pneumologia 28: S81, 2002.

8. Carson FL, Martin JH, Lynn JA. Formalin fixation for electron microscopy. A reevaluation. American Journal of Clinical Pathology 59: 365-373, 1973.

9. Cavallazzi RS, Cavallazzi AC, Souza IV, Cardoso JJ. Dirofilariose pulmonar humana: relato de sete casos. Jornal de Pneumologia 28: 100-102, 2002.

10. Chesney TMcC, Martinez LC, Painter MW. Human pulmonary dirofilarial granuloma. The Annals of Thoracic Surgery 36: 214-217, 1983.

11. Ciferri F. Human pulmonary dirofilariasis in the United States: a critical review. American Journal of Tropical Medicine and Hygiene 31: 302-308, 1982.

12. Coates PJ, D'Ardenne AJ, Khan G, Kangro HO, Slavin G. Simplified procedures for applying the polymerase chain reaction to routinely fixed paraffin wax sections. Journal of Clinical Pathology 44: 115-118, 1991.

13. Cordero M, Munoz MR, Muro A, Simon F. Transient solitary pulmonary nodule caused by Dirofilaria immitis. European Respiratory Journal 3: 1070-1071, 1990.

14. Cordero M, Muro A, Simon F. Pulmonary dirofilariasis. Journal of Thoracic and Cardiovascular Surgery 101: 1110, 1991.

15. Echeverri A, Long RF, Check W, Burnett CM. Pulmonary Dirofilariasis. The Annals of Thoracic Surgery 67: 201-202, 1999.

16. Farber HW, Laguarda R. Human pulmonary dirofilarial infection. Annals of Internal Medicine 106: 777-778, 1987.

17. Favia G, Cancrini G, Ricci I, Bazzocchi C, Magi M, Pietrobelli M, Genchi C, Bandi C. $5 \mathrm{~S}$ ribosomal spacer sequences of some filarial parasites: comparative analysis and diagnostic applications. Molecular and Cellular Probes 14: 285-290, 2000.

18. Fernandes CGN. Aspectos epidemiológicos da dirofilaríase canina no perímetro urbano de Cuiabá, Estado de Mato Grosso, Brasil. Tese de Doutorado, Universidade Federal Rural do Estado do Rio de Janeiro, Rio de Janeiro, RJ, 1999.

19. Gershwin LJ, Gershwin ME, Kritzman J. Human pulmonary dirofilariasis. Chest 66: 92-96, 1974 .

20. Guerra RJA, Oliveira RMF, Almeida FB, Bello AR, Rodrigues-Silva R. Diagnóstico Molecular para Dirofilaríase Pulmonar Humana causada por Dirofilaria immitis baseado na seqüência do espaçador ribossomal ITS2. Jornal de Pneumologia 37 (supl): S81, 2002.

21. Kasai N, Mattos EA, Costa JO. Dirofilaria immitis e Dipetalonema reconditum em cães de Vitória, Espírito Santo. Arquivo da Escola de Veterinária da Universidade Federal de Minas Gerais 33: 425-429, 1981.

22. Knight DH. Heartworm heart disease. Advances in Veterinary Science and Comparative Medicine 21: 107-146, 1977.

23. Lennert K. Malignant Lymphomas other than Hodgkin"s Disease. Springer-Verlag, Berlin, 1978.

24. Leonardi HK, Lapey JD, Ellis Jr H. Pulmonary dirofilariasis: report of a human case. Thorax 32: 612-615, 1977.

25. Madi K, Duarte F, Pignataro PE, Lima RJ, De Paola D. Infartos pulmonares por Dirofilaria. Anais do XVIII Congresso Brasileiro de Patologistas p.79, 1990.

26. Magalhães OS. Descripção de uma espécie de filaria encontrada no coração humano. Revista de Cursos Práticos e Theoricos da Faculdade de Medicina do Rio de Janeiro 3: 129-215, 1887.

27. Narine K, Brennan B, Gilfillan I, Hodge A. Pulmonary presentation of Dirofilaria immitis (canine heartworm) in man. European Journal of Cardiothoracic Surgery 16: 475-477, 1999

28. Nathan MH, Collins VP, Adams RA. Differentiation of being and malignant pulmonary nodules by growth rate. Radiology 79: 221-232, 1962.

29. Pardini MIMC, Carvalho JAB, Gushiken T, Machado PEA. Extração de DNA de tecido emblocado em parafina para utilização em PCR. Journal Brasileiro de Medicina 72: 43-46, 1997 
30. Perkins SL, Kjeldsberg CR. Immunophenotyping of lymphomas and leukemias in paraffin-embedded tissues. American Journal of Clinical Patholology 99: 362-373, 1993.

31. Ro JY, Tsakalakis PJ, White VA, Luna MA, Chang-Tung EG, Green L, Cribbet L, Ayala AG. Pulmonary dirofilariasis: the great imitator of primary or metastatic lung tumor. A clinicopathologic analysis of seven cases and a review of the literature. Human Pathology 20: 69-76, 1989.

32. Robertson ID, Irwin PJ, Lymbery AJ, Thompson RCA. The role of companion animals in the emergence of parasitic zoonoses. International Journal of Parasitology 30: 1369-1377, 2000.

33. Rodrigues-Silva R. Caracterização morfológica e antigênica de Dirofilaria immitis (Leidy, 1856) Railliet \& Henry, 1911. Tese de Doutorado, Fundação Oswaldo Cruz, Rio de Janeiro, RJ, 1996.

34. Rodrigues-Silva R, Moura H, Dreyer G, Rey L. Human Pulmonary Dirofilariasis: a review. Revista do Instituto de Medicina Tropical de São Paulo 37:523-530, 1995.
35. Saad JR, Ethel Filho J, Próspero JD, Dorgan Neto V, Aguiar Neto JR. Dirofilariose pulmonar - relato de um caso. Jornal de Pneumologia 17: 90-93, 1991

36. Schneider CAR, Mirra AP, Justo FA, Oyafuso M, Hidalgo GS, Chieffi PP, Fonseca CAM, Conti RC, Coelho FRG, Soncini L. Dirofilaríase pulmonar humana. Relato de um caso e revisão da literatura. Acta Oncológica Brasileira 6:125-130, 1986.

37. Schubach A, Haddad F, Paes-Oliveira M, Degrave W, Pirmez C, Grimaldi G, Fernandes 0. Detection of Leishmania DNA by Polymerase Chain Reaction in scars of treated human patients. Journal of Infectious Diseases 178: 911-914, 1998.

38. Syed S, Xiao S, Schnadig VJ. Pathologic quiz case. Adolescent girl presenting with solitary pulmonary nodule. Archives of Patholology and Laboratory Medicine 126: 227-228, 2002.

39. Zelch JV, Lalli AF, McCormach LJ, Belovich DM. Aspiration biopsy in diagnosis of pulmonary nodule. Chest 63: 149-152, 1973 